\title{
Sudden death in hypertrophic cardiomyopathy: associated accessory atrioventricular pathways ${ }^{\star}$
}

\author{
DENNIS M KRIKLER, MICHAEL J DAVIES, EDWARD ROWLAND, \\ JOHN F GOODWIN, ROGER C EVANS, DAVID B SHAW
}

From the Division of Cardiovascular Disease, Royal Postgraduate Medical School, Hammersmith Hospital, London; Royal Devon and Exeter Hospital, Exeter; and the Department of Histopathology, St George's Hospital Medical School, London

SUMMARY Sudden death is a known but unpredictable complication of hypertrophic cardiomyopathy. We describe two patients who both had a strong family history of the disorder complicated by sudden death. Necropsy disclosed accessory bypass tracts, concealed in one and previously suspected in the other. One died from ventricular fibrillation and the other, who died outside hospital, had previously complained of palpitation. Arrhythmia complicating pre-excitation appears to be one of the factors responsible for sudden death in hypertrophic cardiomyopathy.

Arrhythmias may be responsible for some cases of sudden death, the most unpredictable and devastating result of hypertrophic cardiomyopathy, and it has been postulated that a proportion of these might occur on the basis of accessory atrioventricular pathways. ${ }^{1}$ Though we demonstrated that concealed pre-excitation ${ }^{2}$ in hypertrophic cardiomyopathy could cause re-entrant atrioventricular tachycardia, ${ }^{1}$ we had no evidence that abnormal atrial activity could reach the ventricles prematurely and initiate ventricular fibrillation, as in some cases of the WolffParkinson-White syndrome. ${ }^{3}$ We now report two patients with hypertrophic cardiomyopathy who died suddenly: they showed identical pathological features, with unusual accessory pathways.

\section{Case reports}

CASE 1

A 17-year-old Yugoslav girl first experienced syncope after exercise at the age of 9 . Subsequently mild exercise produced dyspnoea, light-headedness, exhaustion, and palpitation. There had been six episodes of syncope without chest pain and several attacks suggestive of paroxysmal tachycardia. In July 1976 cardiac catheterisation and angiocardiography in Yugoslavia showed mitral regurgitation and hypertrophy of both ventricles especially *The studies at the Royal Postgraduate Medical School were
supported in part by the British Heart Fo ındation.

Received for publication 12 September 1979 obvious in the interventricular septum, but no intraventricular gradient (details of pressures, and films, are not available). Both her brothers had died suddenly, one at the age of 5 and the other at the age of 15 .

Examination disclosed left ventricular hypertrophy with a palpable atrial beat and a systolic murmur; blood pressure $110 / 70 \mathrm{mmHg}$. The electrocardiogram (Fig. 1) showed sinus rhythm, disturbed atrial depolarisation, first-degree atrioventricular block, moderate left ventricular hypertrophy, and impairment of intraventricular conduction. Chest $x$-ray film showed no evidence of raised left atrial pressure but there was left atrial and ventricular enlargement.

During an intracardiac electrophysiological study ${ }^{4}$ the $\mathrm{AH}$ and $\mathrm{HV}$ intervals were normal at 80 and $45 \mathrm{~ms}$ during sinus rhythm and at the start of atrial pacing (Fig. 2). Incremental right atrial pacing showed 1:1 atrioventricular conduction via the atrioventricular node up to a rate of 187 a minute (Fig. 3) during which the $\mathrm{AH}$ interval did not lengthen but the $\mathrm{HV}$ prolonged slightly $(60 \mathrm{~ms})$. The intraventricular conduction pattern remained unchanged until complex 12; impulses 13 and 14 showed earlier and wider QRS complexes and shorter HV times ( $40 \mathrm{~ms})$, that is earlier ventricular activation. The next stimulus failed to depolarise the ventricles because of atrioventricular block; in complex 15 the HV interval and QRS pattern were as in 1 to 12 , but from 19 onwards the QRS 
complexes were again wide and HV shortened. Stimulation was stopped immediately after complex 26 by which time the patient was in ventricular tachycardia which degenerated into ventricular fibrillation. Though she was promptly defibrillated she was unable to achieve any cardiac output in sinus rhythm or with pacing.

At necropsy, the total heart weight was $510 \mathrm{~g}$, with gross biventricular hypertrophy (Fig. 4). The interventricular septum was asymmetric in relation to the posterior wall of the left ventricle, measuring $2.9 \mathrm{~cm}$ against $1.5 \mathrm{~cm}$ (ratio 1.9:1). The septum bulged into both right and left ventricular outflow tracts. The left ventricular cavity was small $(2 \cdot 1 \mathrm{~cm}$ in diameter at mid-ventricular level). $\mathbf{A}$ band of endocardial fibrous tissue was present on the left side of the interventricular septum forming a mirror image to the anterior cusp of the mitral valve; this finding suggests contact between the septum and anterior cusp during life. On histological examination the myocardium showed the typical malarrangement of hypertrophic cardiomyopathy throughout the whole septum, extending into the free walls of both ventricles. Only in a small lateral segment of the right ventricle and a posterolateral segment of the left ventricle were the muscle fibres normally arranged.

The conduction system and both atrioventricular rings were studied by serial histological section at $120 \mu$ intervals. The sinus node, atrioventricular
Fig. 1 Electrocardiogram of case 1 . Note the presence of distorted $P$ waves, slight $P R$ prolongation, mild left axis deviation, poor $R$ wave development in right praecordial leads, broad $S$ waves in left praecordial leads, and $S T$ depression and/or $T$ wave inversion in leads $I, a V L$, and V5-6.

Fig. 2 Simultaneous intracardiac and surface electrocardiographic recordings in case 1. The arrows, and corresponding artefacts, indicate right atrial stimulation. In addition to leads $I, I I I, V 1$, and V6, tracings were recorded from the coronary sinus (CSE), reflecting activity from the left atrium ( $L A)$; the low right atrium and ventricle ( $H B E$, His bundle electrogram); and right ventricle $(R V)$. LRA, low right atrial depolarisation; $H$, activation of bundle of $\mathrm{His}$; $V$, ventricular activation. Paper speed $100 \mathrm{~mm} / \mathrm{s}$.
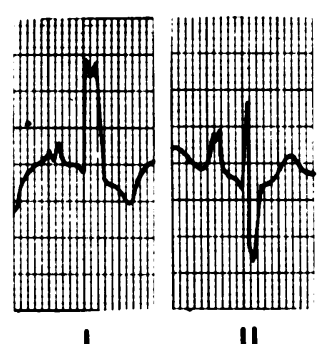

I

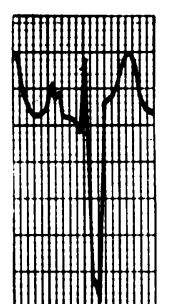

III

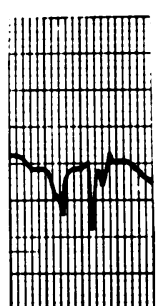

aVR

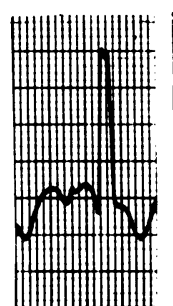

aVL
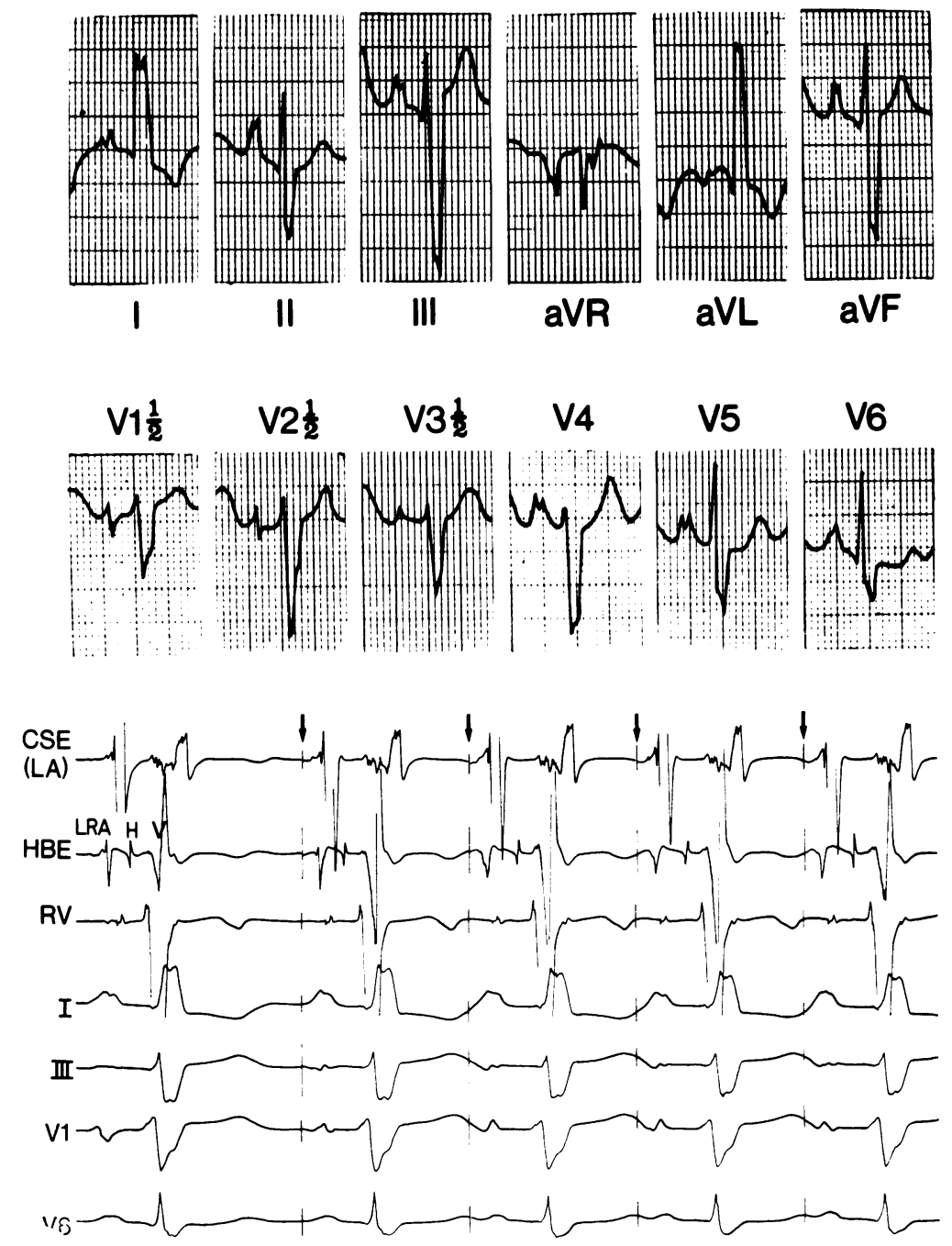


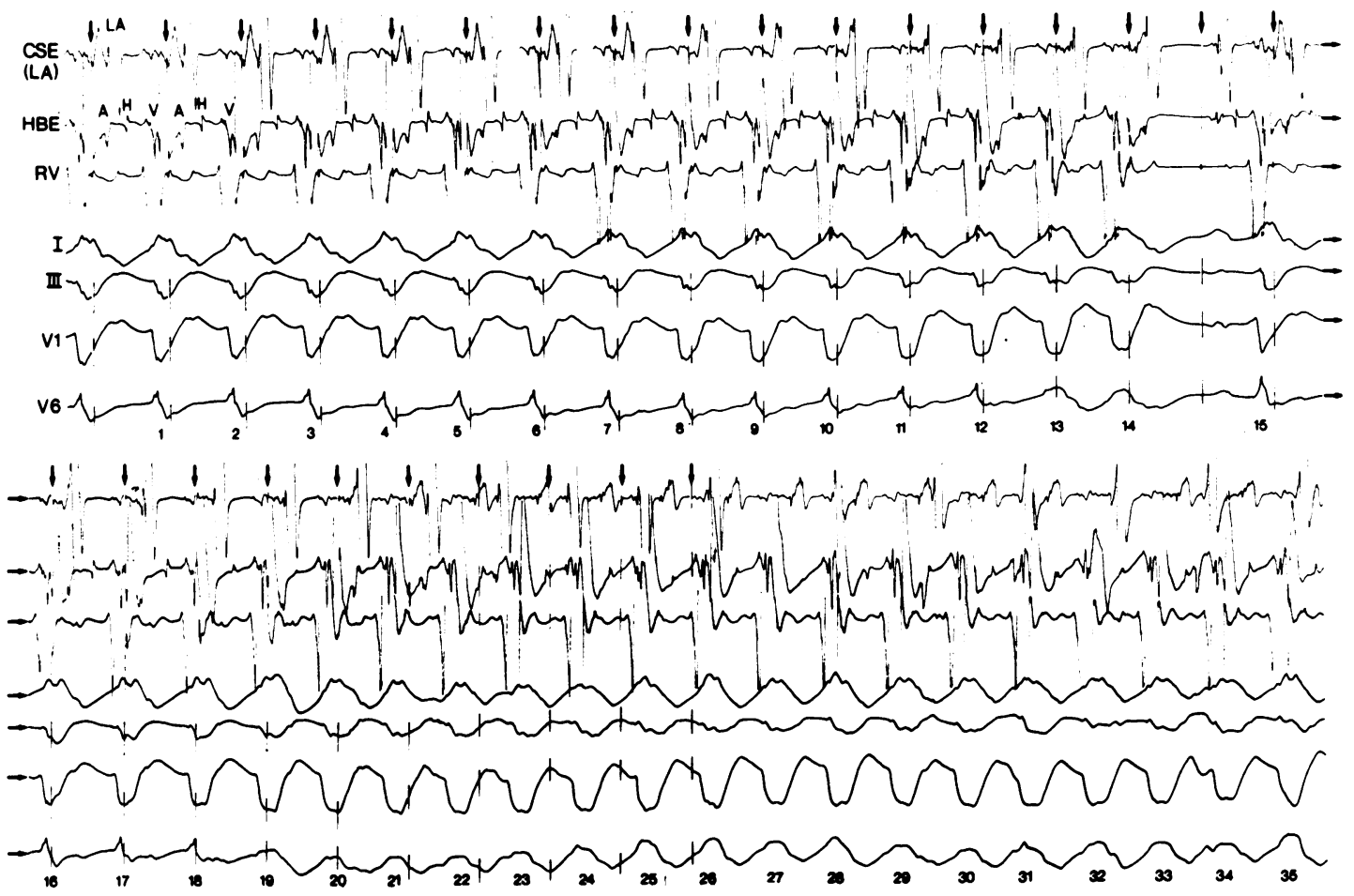

Fig. 3 Recordings as in Fig. 2, with incremental right atrial pacing. Each stimulus is followed by ventricular activation with consistent $Q R S$ morphology until complexes 13 and 14, when $Q R S$ is broader and ventricular activation earlier; $2: 1$ atrioventricular block follows complex 14, complexes 15 to 18 resembling earlier patterns. From 19 onwards, QRS is widened and ventricular depolarisation earlier; after 26 there is independent ventricular tachycardia.

node, penetrating and bifurcating main bundle, and proximal bundle-branches were within normal morphological limits. On the right side of the interventricular septum, muscle bands passed up under the septal cusp of the tricuspid valve and, because of fragmentation of the collagen of the central fibrous body, made multiple connections with atrial muscle fibres running immediately beneath the right atrial endocardium superficial to the atrioventricular node (Fig. 5). These atrioventricular connections occurred in an area $0.5 \mathrm{~cm}$ in length and were composed at the ventricular end of hypertrophic disorganised muscle which extended anteriorly close to the right bundle-branch.

\section{CASE 2}

A 28-year-old woman had been found at the age of 5 to have a systolic murmur which was initially attributed to a ventricular septal defect. The electrocardiogram then was said to show left bundlebranch block; later the PR interval was seen to be short and Wolff-Parkinson-White syndrome was suspected. She had no cardiac symptoms until the

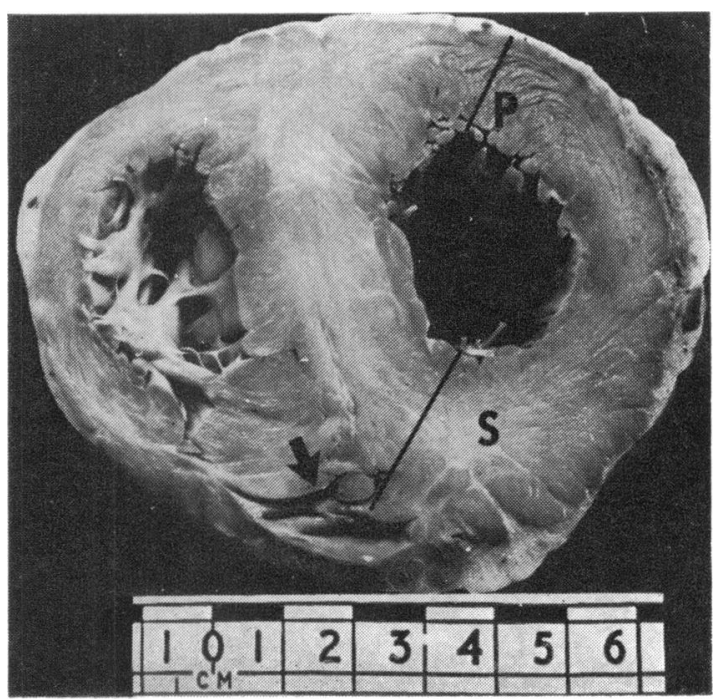

Fig. 4 Transverse section of left and right ventricles of case 1. The anterior portion of the interventricular septum $(S)$ is disproportionately thickened in comparison with the posterior left ventricular wall $(P)$. The septum bulges into the right ventricular outflow tract (arrow). 
menarche (age 13) when she developed palpitation, aggravated by effort, before each period. At the age of 18 the cardiac apex was normal, there was an early aortic systolic murmur, grade $3 / 6$; blood pressure $160 / 80 \mathrm{mmHg}$, and electrocardiogram unchanged (Fig. 6a). Practolol $100 \mathrm{mg}$ twice daily had little effect but propranolol appeared to reduce the number of attacks. Dyspnoea on effort first developed at the age of 24 . The electrocardiogram now showed even greater PR shortening, and wider QRS complexes with increased voltage and smaller

Fig. 5 Histological sections of heart (case 1). (a) The plane of section passes through the atrial and ventricular septum immediately posterior to the atrioventricular node. Adjacent to the mitral valve (M), atrial $(A)$ and ventricular $(V)$ muscle are clearly separated by the fibrous body. Adjacent to the tricuspid valve (T) a mass of muscle (arrows) from the ventricular septum pushes up under the septal cusp and the fibrous body is indistinct. (Van Gieson. Original magnification $\times 14$.) (b) Area adjacent to the mitral valve in (a). Atrial muscle $(A)$ is separated from ventricular muscle (V) by a distinct mass of fibrous tissue. (Van Gieson. $\times 100$.) (c) Area adjacent to the tricuspid valve in (a). Multiple strands of muscle (arrow) pass from atrial ( $A$ ) to ventricular $(V)$ muscle; no defined fibrous body is present. (Haematoxylin and eosin. $\times 100$.)
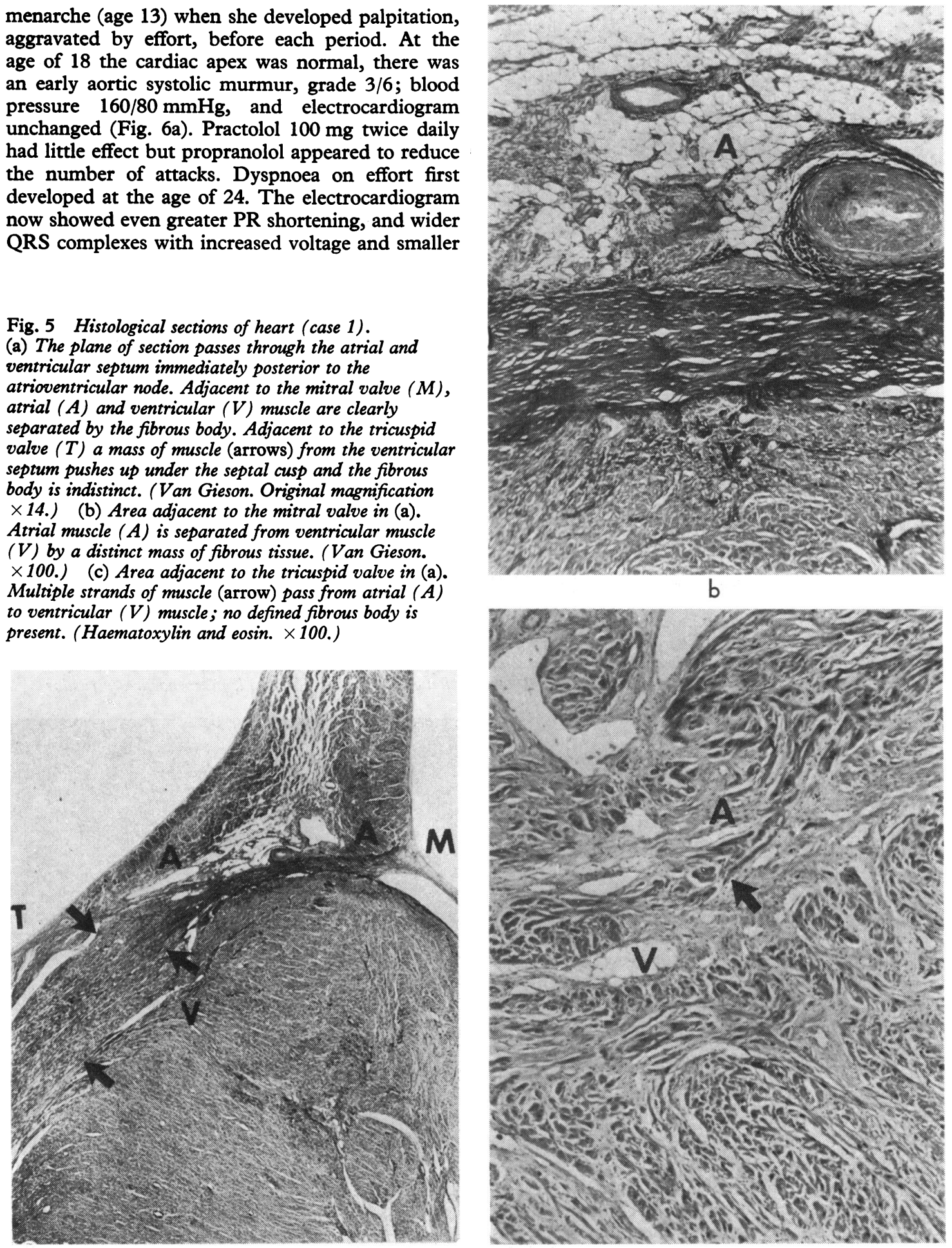

C 
$\mathbf{R}$ waves in right praecordial leads (Fig. 6b). Electrophysiological investigations were considered but deferred; three months later she collapsed while clearing away snow and was dead on arrival at hospital.

Her father had died suddenly at the age of 31 , necropsy showing pronounced hypertrophy of both ventricles, the left more than the right (heart weight $575 \mathrm{~g}$ ). A younger sister died suddenly at the age of 9: the heart weighed $300 \mathrm{~g}$ and there was 'moderate degree of generalised hypertrophy' without outflow tract obstruction. Her only brother is 27 , alive, symptom-free, and without clinical or electrocardiographic abnormality.

At necropsy, the total heart weight was $560 \mathrm{~g}$, with biventricular hypertrophy. The interventricular septum measured $4 \mathrm{~cm}$ and the posterior left ventricular wall $2 \cdot 2 \mathrm{~cm}$ (ratio 1.8:1). The left ventricular cavity was small $(2 \mathrm{~cm}$ at midventricular level). Abnormally arranged muscle was present in the majority of both ventricles, only sparing a small posterior segment of each. Involvement of the right ventricular free wall in the outflow produced anatomical obstruction.

The conduction system and the whole of both atrioventricular rings were examined by serial section. The atrioventricular node, main bundle, and proximal bundle-branches were normal. The left bundle-branch in the mid-septal zone showed some attenuation and stretching with distinct endocardial fibrosis. In the right atrioventricular groove, anteriorly over an area of $3 \mathrm{~mm}$, a band of subepicardial atrial fibres made connection with a mass of ventricular fibres (Fig. 7) that showed the typical arrangement of hypertrophic cardiomyopathy. A second small atrioventricular connection was present in the interventricular septum in the identical position to that of case 1 . This connection, consisting of a single muscle bundle $20 \mu$ across, joined abnormal muscle in the interventricular septum to the atrial fibres passing down superficial to the atrioventricular node.

\section{Discussion}

Both patients were young women who had symptoms and a strong family history of sudden death. In the first patient ventricular fibrillation followed atrial stimulation during an electrophysiological study; the other had previously been shown to have electrocardiographic abnormalities consistent with pre-excitation. The electrophysiological study was performed in the first patient to see whether means could be found to prevent sudden death, the major hazard to which she was liable. At necropsy both patients were found to have hypertrophic cardiomyopathy and identical and unusual accessory atrioventricular connections made up of the same type of tissue as was found in the ventricles. Though mechanisms other than arrhythmias undoubtedly produce sudden death in some patients, ${ }^{1}$ and other arrhythmias, for example ventricular tachycardia, may occur, ${ }^{56}$ our hypothesis has been substantiated as far as these two patients are concerned. ${ }^{1}$ Further investigation is required to see how common this phenomenon is and whether one can prevent this outcome.

The possibility that, in case 1 , there was enhanced atrioventricular conduction through the node seems to us a much less reasonable explanation in the face of the anatomical abnormality, and because the alteration in QRS morphology is consistent with stimulation of the right ventricle through an accessory pathway in that particular location. This a)
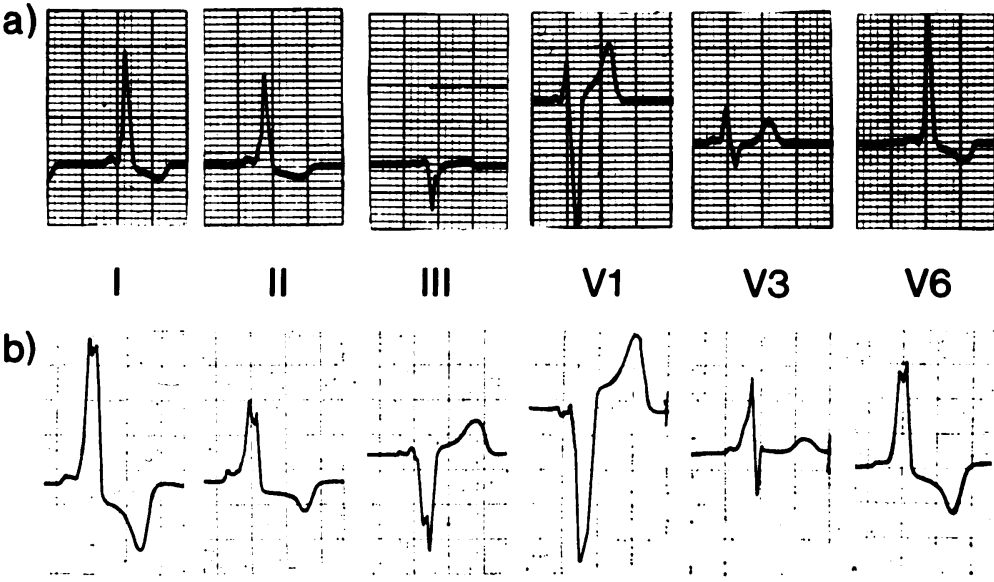

V3

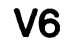

V6

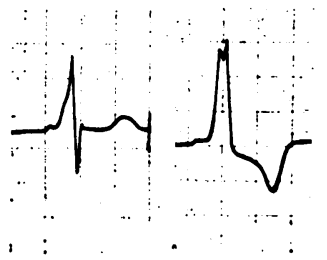

Fig. 6 Electrocardiograms of case 2 (a) in early childhood and (b) three months before death. In both there are short $P R$ intervals and wide $Q R S$ complexes, but the latter tracings show greater widening and distortion of the $Q R S$ complexes and more obvious $S T-T$ depression and inversion, with loss of the $R$ wave in V1. In both instances, the upstroke of $R$ is slurred, resembling a delta wave. 
is supported by the fact that, after resumption of atrioventricular conduction at complex 15, the QRS morphology reverted to its previous appearance; the early ventricular activation is of crucial importance and, we believe, most consistent with the anatomical findings.

Patients with hypertrophic cardiomyopathy who die suddenly are often children and young adults, and there may be a relation to physical exertion; in addition there are electrocardiographic abnormalities including increasing left ventricular hypertrophy and pronounced ventricular septal thickening. ${ }^{7}$ This may occur in families who may show an unusually severe expression of the disorder. ${ }^{8}$ Abnormal accessory atrioventricular communications were not reported in these cases, ${ }^{7}$ though septal clefts have been considered to be responsible. ${ }^{9}$ We did not think that such changes could explain the electrophysiological abnormalities found in patients suffering from re-entrant atrioventricular tachycardia caused by concealed pre-excitation in

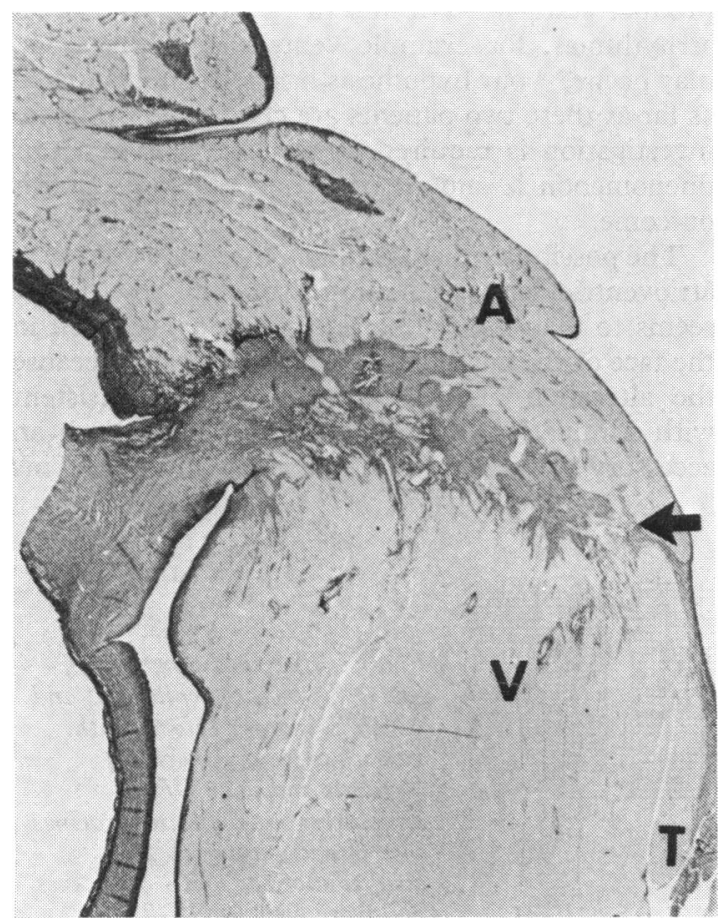

Fig. 7 Section through the heart of case 2, passing through atrial $(A)$ and ventricular $(V)$ septa immediately posterior to the atrioventricular node. The central fibrous body is penetrated by a mass of muscle (arrow) extending under the tricuspid valve ( $T$ ) and connecting atrial and ventricular septa. (Van Gieson. $\times 10$.) hypertrophic cardiomyopathy, ${ }^{1}$ and had proposed to investigate the electrophysiological characteristics of patients at risk of sudden death with a view to finding factors that might prevent this.

Though it has been suggested that pre-excitation and hypertrophic cardiomyopathy frequently coexist, a review of published cases showed that this association was infrequent ${ }^{10}$; we felt that in many the appearances were the result of a combination of shortening of the PR interval and abnormal intraventricular conduction as well as hypertrophy. ${ }^{1}$ Careful electrophysiological study of 13 patients with proven hypertrophic cardiomyopathy showed a high incidence of arrhythmias and conduction abnormalities, particularly within the His-Purkinje system, ${ }^{11}$ though we think it will be necessary to show whether similar findings might occur in patients with left ventricular hypertrophy of similar degree but resulting from other causes. In none of the 13 cases were there signs of pre-excitation though atrioventricular nodal behaviour suggested a possible tendency for the development of intranodal reciprocating tachycardias. ${ }^{11}$

We have not carried out further invasive electrophysiological investigations on patients with highrisk cardiomyopathy as judged by the family history and findings thought to suggest the likelihood of sudden death. ${ }^{6}{ }^{12}$ As patients with this disease are notoriously difficult to resuscitate from ventricular fibrillation, doubtless because of the grossly diseased myocardium, such studies should probably be avoided in high-risk patients at this stage of our knowledge.

The electrocardiogram in the first patient showed no evidence of pre-excitation while that in the second did suggest the Wolff-Parkinson-White syndrome, though probably, at least latterly, modified by left bundle-branch block. ${ }^{13}$ The location of the bypass in the first case is consistent with the electrophysiological findings; when right atrial stimuli blocked the atrioventricular node and engaged the bypass, the QRS complexes were premature and resembled left bundle-branch block because the earliest part of the ventricles to be excited was the right upper interventricular septum. Pre-excitation was also shown by unduly rapid transmission of atrial impulses to the ventricles ${ }^{14}$ and HV shortening, ${ }^{4}$ both of which reflect the absence of normal atrioventricular nodal delaying properties. In the second case there were two bypasses; it is impossible, without electrophysiological study, to know which was implicated. ${ }^{10}$ It is more likely that the abnormal tissue found in hypertrophic cardiomyopathy invaded pre-existing latent accessory pathways ${ }^{2}$ rather than eroding the annulus fibrosus or central fibrous body. 


\section{References}

${ }^{1}$ Goodwin JF, Krikler DM. Arrhythmia as a cause of sudden death in hypertrophic cardiomyopathy. Lancet 1976; 2: 937-40.

${ }^{2}$ Krikler DM, Rowland E. Concealed pre-excitation. F Electrocardiol 1978; 11 : 209-11.

${ }^{3}$ Dreifus LS, Haiat R, Watanabe Y, Arriaga J, Reitman NC. Ventricular fibrillation, a possible mechanism of sudden death in patients with the Wolff-ParkinsonWhite syndrome. Circulation 1971; 43: 520-7.

${ }^{4}$ Krikler DM. A fresh look at cardiac arrhythmias. Electrocardiographic diagnosis. Lancet 1974; 1: 974-6. ${ }^{5}$ McKenna WJ, Chetty S, Oakley CM, Goodwin JF. Exercise electrocardiographic and 48-hour ambulatory electrocardiographic monitor assessment of arrhythmia on and off beta-blocker therapy in hypertrophic cardiomyopathy (abstract). $B r$ Heart $f$ 1979; 41: 375-6.

${ }^{6}$ Savage DD, Seides SF, Maron BJ, Myers DJ, Epstein SE. Prevalence of arrhythmias during 24-hour electrocardiographic monitoring and exercise testing in patients with obstructive and non-obstructive hypertrophic cardiomyopathy. Circulation 1979; 59: 866-75.

${ }^{7}$ Maron BJ, Roberts WC, Edwards JE, McAllister HA Jr, Foley DD, Epstein SE. Sudden death in patients with hypertrophic cardiomyopathy: characterisation of 26 patients without functional limitation. Am ₹ Cardiol 1978; 41: 803-10.

${ }^{8}$ Maron BJ, Lipson LC, Roberts WC, Savage DD, Epstein SE. 'Malignant' hypertrophic cardiomyopathy: identification of a subgroup of families with unusually frequent premature death. Am $f$ Cardiol 1978; 41: 1133-40.

${ }^{9}$ James TN, Marshall TK. De subitaneis mortibus XII Asymmetrical hypertrophy of the heart. Circulation 1975; 51: 1149-66.

${ }^{10}$ Krikler DM. The Wolff-Parkinson-White and related syndromes. I. Presentations and implications. In: Krikler DM, Goodwin JF, eds. Cardiac arrhythmias, the modern electrophysiological approach. London, Philadelphia, and Toronto: WB Saunders, 1975: 144-61.

${ }^{11}$ Ingham RE, Mason JW, Rossen RM, Goodman DJ, Harrison DC. Electrophysiologic findings in patients with idiopathic hypertrophic subaortic stenosis. $A m f$ Cardiol 1978; 41: 811-6.

${ }^{12}$ Swan DA, Bell B, Oakley CM, Goodwin JF. Analysis of symptomatic course and prognosis and treatment of hypertrophic obstructive cardiomyopathy. $\mathrm{Br} \mathrm{Heart} \mathcal{f}$ 1971; 33: 671-85.

${ }^{13}$ Krikler D, Coumel Ph, Curry P, Oakley C. WolffParkinson-White syndrome type A obscured by left bundle branch block. Eur f Cardiol 1977; 5: 49-62.

${ }^{14}$ Krikler DM, Curry PVL. The paroxysmal supraventricular tachyarrhythmias. In Yu PN, Goodwin JF, eds. Progress in cardiology 5. Philadelphia: Lea \& Febiger, 1976: 291-319.

Requests for reprints to $\mathrm{Dr}$ Dennis Krikler, Division of Cardiovascular Disease, Royal Postgraduate Medical School, Hammersmith Hospital, London W12 0HS. 